\title{
Repeatability and responsiveness of exercise tests in pulmonary arterial hypertension
}

\author{
Vincent Mainguy, Simon Malenfant, Anne-Sophie Neyron, Sébastien Bonnet, \\ François Maltais, Didier Saey and Steeve Provencher
}

Affiliation: Pulmonary Hypertension Research Group, Centre de recherche de l'Institut Universitaire de cardiologie et de pneumologie de Québec, Université Laval, Québec, QC, Canada.

Correspondence: S. Provencher, Centre de recherche de l'Institut Universitaire de cardiologie et de pneumologie de Québec, Service de Pneumologie, 2725, chemin Sainte-Foy, Québec, QC, G1V 4G5, Canada. E-mail: steve.provencherdcriucpq.ulaval.ca

ABSTRACT Exercise tolerance in pulmonary arterial hypertension (PAH) is most commonly assessed by the 6-min walk test $(6 \mathrm{MWT})$. Whether endurance exercise tests are more responsive than the $6 \mathrm{MWT}$ remains unknown.

20 stable $\mathrm{PAH}$ patients (mean $\pm \mathrm{SD}$ age $53 \pm 15$ years and mean pulmonary arterial pressure $44 \pm 16 \mathrm{mmHg}$ ) already on PAH monotherapy completed the 6MWT, the endurance shuttle walk test (ESWT) and the cycle endurance test (CET) before and after the addition of sildenafil citrate $20 \mathrm{mg}$ three times daily or placebo for 28 days in a randomised double-blind crossover setting. Pre- or post-placebo tests were used to assess repeatability of each exercise test, whereas pre- or post-sildenafil citrate tests were used to assess their responsiveness.

Sildenafil citrate led to placebo-corrected changes in exercise capacity of $18 \pm 25 \mathrm{~m}(\mathrm{p}=0.02), 58 \pm 235 \mathrm{~s}$ $(\mathrm{p}=0.58)$ and $29 \pm 77 \mathrm{~s}(\mathrm{p}=0.09)$ for the 6MWT, the ESWT and the CET, respectively. The 6MWT was associated with a lower coefficient of variation between repeated measures ( $3 \%$ versus $18 \%$ versus $13 \%$ ), resulting in a higher standardised response mean compared with endurance tests $(0.72,0.25$ and 0.38 for the $6 \mathrm{MWT}$, the ESWT and the CET, respectively).

The 6MWT had the best ability to capture changes in exercise capacity when sildenafil citrate was combined with patients' baseline monotherapy, supporting its use as an outcome measure in PAH.

@ERSpublications

Exercise tests in PAH: the 6MWT has a better test-retest repeatability and responsiveness compared with endurance tests http://ow.ly/lB2wY

Received: July 112012 | Accepted after revision: Oct 042012 | First published online: Oct 252012

Clinical Trial: This study is registered at www.clinicaltrials.gov with identifier number NCT01391104.

Support statement: Vincent Mainguy is funded by the Fonds de Recherche du Québec - Santé (grant number 20701).

Conflict of interest: Disclosures can be found alongside the online version of this article at www.erj.ersjournals.com

Copyright @ERS 2013 


\section{Introduction}

Pulmonary arterial hypertension (PAH) is characterised by the progressive increase in pulmonary vascular resistance leading to right heart failure, poor exercise tolerance and early death. Because of its relationship with functional class and survival, exercise capacity is generally considered as a surrogate marker of disease severity in PAH [1]. As a result, the 6-min walk test (6MWT) has been the primary end-point in the majority of the clinical trials [2].

In PAH, the 6MWT has been assumed to be repeatable and more responsive to clinical changes than the incremental cardiopulmonary exercise test (CPET) on ergocycle [3]. Based on these results, the 6MWT has become, by far, the most popular exercise testing modality in $\mathrm{PAH}$ [2]. However, the repeatability of the 6MWT has never been formally assessed in PAH, and its responsiveness to therapeutic intervention is questionable. Indeed, recent meta-analyses documented that the increase in 6MWT following monotherapy and combination therapy averaged $35 \mathrm{~m} \mathrm{[2]} \mathrm{and} 22 \mathrm{~m} \mathrm{[4],} \mathrm{respectively,} \mathrm{corresponding} \mathrm{to} \sim 10 \%$ and $\sim 7 \%$ of baseline values. Moreover, the 6MWT may suffer from a ceiling effect in patients with mild PAH [5]. These observations suggest that the responsiveness of the 6MWT to interventions may be insufficient in $\mathrm{PAH}$, leading to underestimation of the real treatment benefit and potentially to impeding drug development in this disease. The validation of a reliable exercise testing modality that is repeatable and responsive to interventions is therefore clearly needed in PAH. The endurance shuttle walk test (ESWT) and the cycle endurance test (CET) have been used in other chronic diseases, such as chronic obstructive pulmonary disease (COPD), and were shown to be more sensitive to clinical changes than the 6MWT or the CPET $[6,7]$. CET is now the primary end-point in most controlled trials evaluating bronchodilators and exercise training in COPD. Conversely, endurance exercise tests have been rarely used in PAH and their responsiveness remains unknown.

The objective of this study was to compare the test-retest repeatability and responsiveness of the 6MWT, the ESWT and the CET in PAH. We hypothesised that the endurance tests would be as repeatable as the $6 \mathrm{MWT}$, whereas their responsiveness following the addition of sildenafil citrate over initial monotherapy would be superior to that of the 6MWT.

\section{Methods}

\section{Ethics statement}

The institutional ethics committee (Comité d'éthique de la recherche de l'Institut Universitaire de cardiologie et de pneumologie de Québec, Université Laval, Québec, QC, Canada) approved the research protocol (protocol number CÉR 20414) and all patients gave written consent prior to study enrolment.

\section{Subjects}

Consecutive PAH patients on monotherapy but naive to phosphodiesterase type- 5 inhibitors were recruited at the Institut Universitaire de cardiologie et de pneumologie de Québec (Université Laval, Québec). PAH diagnosis was made according to recent guidelines [8]. Recent right heart catheterisation $(<6$ months previously) was used to describe haemodynamic severity. Only patients with stable clinical condition and medication over the last 4 months were eligible. Exclusion criteria were as follows. 1) Unstable PAH defined as recent syncope or World Health Organization (WHO) functional class IV; 2) left ventricular ejection fraction $<40 \%$; 3 ) significant restrictive (more than minimal lung fibrosis on computed tomography scan or total lung capacity $<70 \%$ of predicted) or obstructive (forced expiratory volume in $1 \mathrm{~s} /$ forced vital capacity $<70 \%$ ) lung disease; and 4) systemic blood pressure $<100 / 60 \mathrm{mmHg}$.

\section{Study design}

The design of this randomised, double-blind, crossover study is shown in figure 1 .

\section{Familiarisation phase (visits 1 and 2)}

On day 1, a CPET was performed on an electrically braked ergocycle (Corival, Lode B.V., Groningen, the Netherlands) [9]. After $3 \mathrm{~min}$ of rest and $1 \mathrm{~min}$ of unloaded pedalling, patients exercised using an individualised progressive ramp protocol until exhaustion. Dyspnoea and leg fatigue were assessed at the end of the test using a 10-point modified Borg scale [10]. Patients then performed a practice CET. After 1 min of unloaded pedalling, the workload was set at $80 \%$ of peak workload achieved during the CPET. Patients were told to pedal for as long as possible at a minimum rate of $60 \mathrm{rpm}$ until exhaustion. The endurance time was defined as the total exercise duration, excluding unloaded pedalling.

On day 2, a 6MWT was performed on a flat 30-m course according to recommendations [11]. An incremental shuttle walk test (ISWT) was also performed in an enclosed corridor on a flat 10-m course delimited by two cones positioned $0.5 \mathrm{~m}$ from either end [12]. Patients had to follow the rhythm dictated 

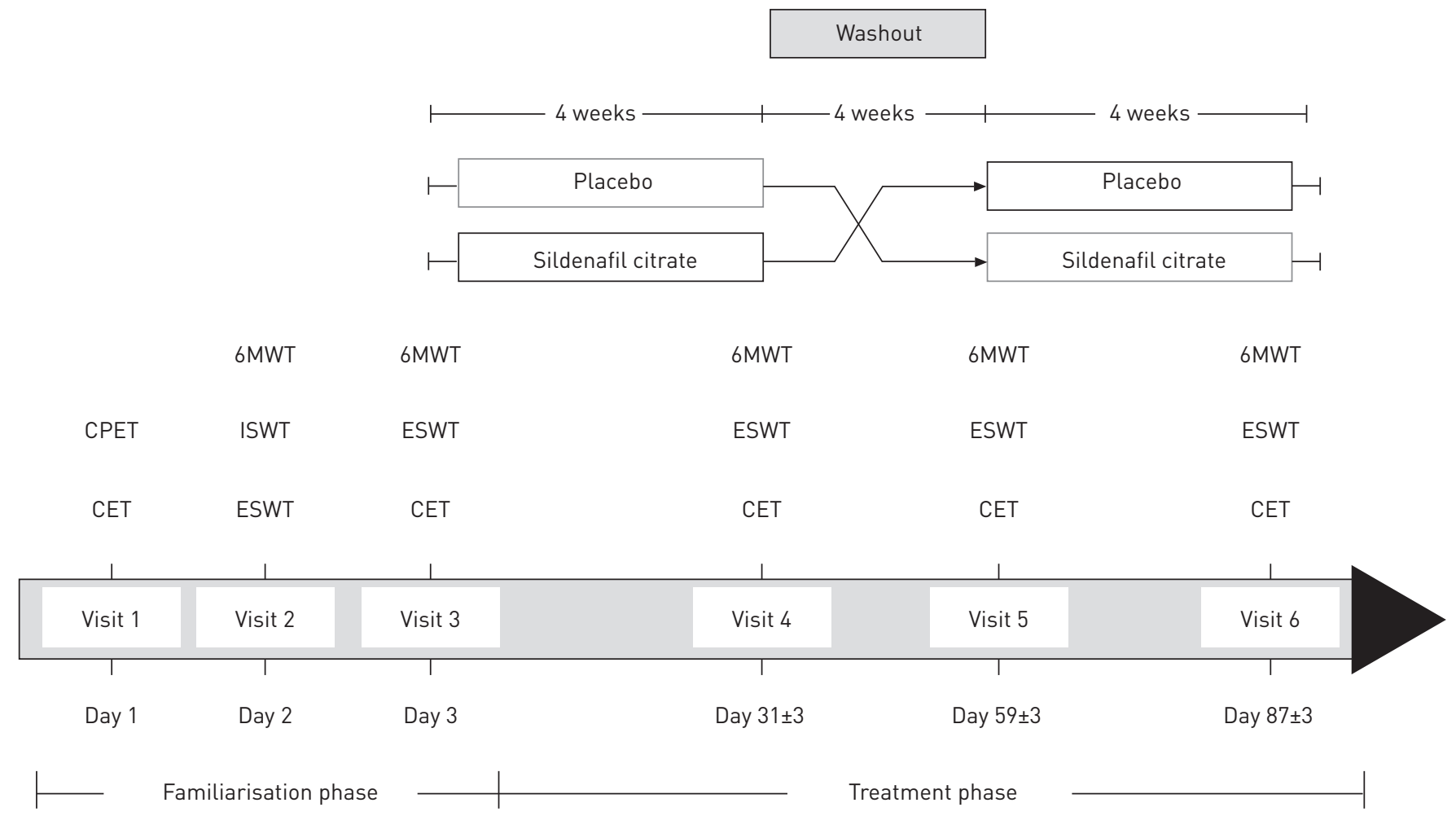

FIGURE 1 Study design. During the first two visits, patients performed an incremental cardiopulmonary exercise test (CPET) and an incremental shuttle walk test (ISWT). A practice 6-min walk test (6MWT), endurance shuttle walk test (ESWT) and cycle endurance test (CET) were also performed as part of a familiarisation phase. On visit 3, patients performed baseline 6MWT, ESWT and CET before they received sildenafil citrate or a matching placebo of identical appearance in a double-blind manner for 28 days. Patients were re-evaluated at visit 4, keeping the same test order throughout the study. After a 4-week washout period, the 6MWT, ESWT and CET were performed again before and after $28 \pm 3$ days of the alternate therapy (visits 5 and 6 ).

by an audio signal. The initial walking speed was set at $0.5 \mathrm{~m} \cdot \mathrm{s}^{-1}$ and subsequently increased every minute by $0.17 \mathrm{~m} \cdot \mathrm{s}^{-1}$ until exhaustion. Finally, a practice ESWT was performed on the same flat 10-m course [13]. Following a 1.5-min warm-up period, the walking speed was set at $85 \%$ of the peak walking speed achieved during the ISWT. The rhythm was dictated by an audio signal. The tests were performed $2 \mathrm{~h}$ apart.

Treatment phase (visits 3-6)

At visit 3, the 6MWT, ESWT and CET were performed $2 \mathrm{~h}$ apart [9]. To minimise any confounding effect based on test sequence, the order of the tests for each subject was randomly determined using the Latin square design. Each patient then kept his individualised order for subsequent visits. Following this visit, patients were randomised to receive sildenafil citrate $20 \mathrm{mg}$ three times daily or a matching placebo in a double-blind manner for 28 days. To ensure safety, patients were asked to measure their systemic blood pressure once a day during the first 3 days under both treatments. Blood pressure measurements were reported to a research nurse and were not reported to the study physician. Treatment was stopped in case of persistent headaches, systemic hypotension $(<100 / 60 \mathrm{mmHg})$ or any significant side-effect. Patients were re-evaluated at visit 4 , after $28 \pm 3$ days of treatment with sildenafil citrate or placebo, in a similar manner to visit 3. Then, after a 4 -week washout period, the $6 \mathrm{MWT}$, ESWT and CET were performed again before and after $28 \pm 3$ days of the alternate therapy (visits 5 and 6). Pre- or post-placebo tests were used to assess the repeatability of each exercise test, whereas pre- or post-sildenafil citrate tests were used to assess the capacity of each test to detect changes in exercise capacity following PAH pharmacological treatment.

\section{Physiological monitoring and measurements during exercise tests}

During all exercise tests, cardiac parameters using a 12-lead ECG, breath-by-breath respiratory parameters and pulse oximetry were continuously monitored with a portable telemetric device (Oxycon Mobile, Viasys Healthcare, Hoechberg, Germany). While encouragements were provided throughout incremental tests, no verbal encouragements were made during the 6MWT and endurance tests, as recommended [9, 11]. Patients finally rated their perception of exercise performance after each treatment phase using a 7-point Likert scale. This scale ranged from -3 to +3 and included the following ratings: -3 (large deterioration), 
-2 (moderate deterioration), -1 (slight deterioration), 0 (no change), 1 (slight improvement), 2 (moderate improvement) and 3 (large improvement).

\section{Statistical analysis}

The main outcome of interest was the capacity of each test to detect a significant improvement following the addition of sildenafil citrate to the current monotherapy. The primary outcome was thus the placebocorrected change in exercise duration/length for each exercise test. Patients without post-baseline assessments were excluded from this analysis. A crossover statistical analysis, corrected for baseline values (covariable) was used. Repeatability of each exercise test was assessed using Pearson correlations and coefficients of variation between pre/post-placebo results. The coefficients of variation were calculated by dividing the standard deviation of the change by the mean change of exercise duration/length between postplacebo versus pre-placebo and multiplied by 100 [14]. A modified Bland-Altman plot showing the relative change from mean according to the mean duration/length of the test was also used to appreciate the limits of agreement for each exercise test. The standardised response mean of each test was also computed as a marker of both the responsiveness and repeatability. This parameter was obtained by dividing the mean placebo-corrected difference in the exercise capacity between baseline and post-treatment by the standard deviation of the change of the corresponding exercise modality (magnitude of change/standard deviation of change).

In secondary analyses, the repeatability/responsiveness of the physiological response during the 6MWT, the ESWT and the CET at peak exercise were compared. Pearson correlations were performed to explore the relationship between the changes in exercise capacity captured by each exercise test and the Likert scores using post-treatment and post-placebo data (two measures per patient). For each exercise test, we constructed a receiver operating characteristic (ROC) curve at progressive changes in exercise capacity to estimate the optimal threshold for both specificity and sensitivity to detect improvement of $\geqslant 1$ on the Likert score. The multivariate normality assumption was verified with the Shapiro-Wilk test after Cholesky factorisation. Univariate normality and variance assumptions were verified using the Shapiro-Wilk and Brown-Forsythe tests, respectively. The results are expressed as mean \pm SD unless specified otherwise. A statistical level of significance of $\mathrm{p}=0.05$ was used. All analyses were run blinded using SAS statistical software, version 9.2 (SAS Institute Inc., Cary, NC, USA). The sample size was estimated using the 6MWT. Assuming a mean placebo-corrected difference in $6 \mathrm{MWT}$ of $25 \pm 45 \mathrm{~m}$ between baseline and post-treatment and a drop-out rate of $20 \%, 25$ subjects were necessary to detect a significant treatment effect with a type I error of $5 \%$ and a type II error of $20 \%$.

\section{RESULTS}

\section{Patients' characteristics}

A total of 22 patients were recruited from June 2009 to August 2011. We excluded one patient during the familiarisation phase due to a significant ST segment depression during exercise and another patient withdrew consent during the second treatment phase (fig. 2). Baseline characteristics of the $20 \mathrm{PAH}$ patients who completed the entire protocol are shown in table 1. Most patients had idiopathic PAH or PAH

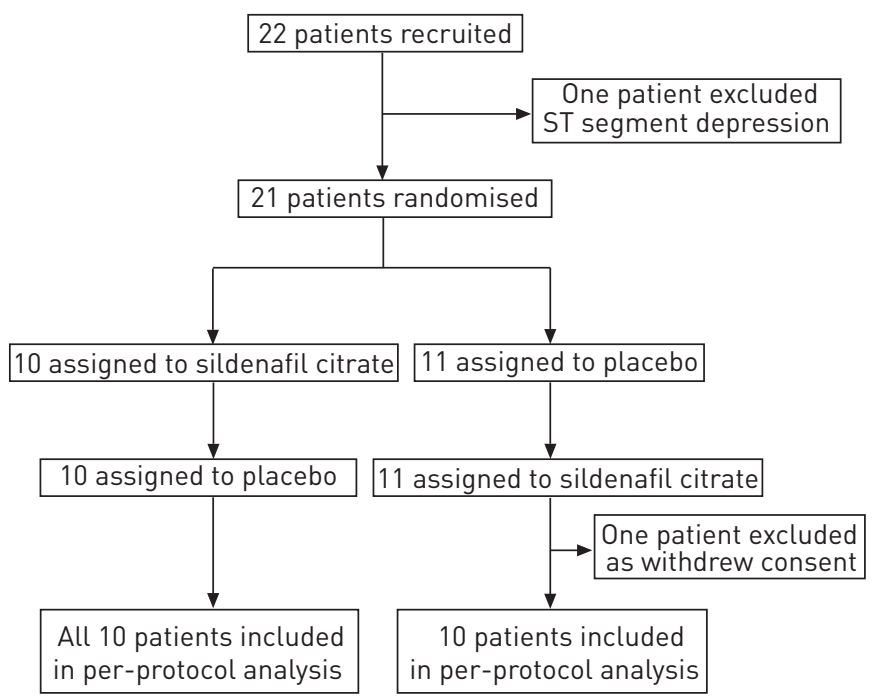

FIGURE 2 Study flow chart. 


\section{TABLE 1 Patient characteristics}

\begin{tabular}{|c|c|}
\hline Subjects & 20 \\
\hline \multicolumn{2}{|l|}{ PAH type } \\
\hline IPAH & 9 \\
\hline $\mathrm{PAH}-\mathrm{Her}$ & 1 \\
\hline PAH-CTD & 8 \\
\hline $\mathrm{PAH}-\mathrm{CHD}^{\#}$ & 2 \\
\hline Sex female/male & $16 / 4$ \\
\hline Age years & $53 \pm 15$ \\
\hline $\mathrm{BMI} \mathrm{kg} \cdot \mathrm{m}^{-2}$ & $27 \pm 5$ \\
\hline WHO functional class II/III & $15 / \overline{5}$ \\
\hline \multicolumn{2}{|l|}{ Pulmonary haemodynamics } \\
\hline $\mathrm{RAP} \mathrm{mmHg}$ & $6.4 \pm 3.6$ \\
\hline Mean PAP $\mathrm{mmHg}$ & $44 \pm 16$ \\
\hline PCWP $\mathrm{mmHg}$ & $10 \pm 4$ \\
\hline Cardiac index $\mathrm{L} \cdot \mathrm{min}^{-1} \cdot \mathrm{m}^{-2}$ & $3.0 \pm 0.6$ \\
\hline PVRI WU $\cdot \mathrm{m}^{-2}$ & $6.6 \pm 3.1$ \\
\hline 6MWD m & $448 \pm 98$ \\
\hline 6MWD \% pred & $78 \pm 15$ \\
\hline \multicolumn{2}{|l|}{ CPET } \\
\hline HR beats $\cdot \min ^{-1}$ & $138 \pm 24$ \\
\hline$V^{\prime} \mathrm{O}_{2} \mathrm{~mL} \cdot \mathrm{min}^{-1}$ & $1008 \pm 303$ \\
\hline$V^{\prime} \mathrm{O}_{2} \mathrm{~mL} \cdot \mathrm{kg}^{-1} \cdot \mathrm{min}^{-1}$ & $14.65 \pm 4.18$ \\
\hline RER & $1.23 \pm 0.12$ \\
\hline$V^{\prime} \mathrm{E} L \cdot \min ^{-1}$ & $59 \pm 9$ \\
\hline $\mathrm{SpO}_{2} \%$ & $89 \pm 6$ \\
\hline$V^{\prime} \mathrm{E} / \mathrm{V}^{\prime} \mathrm{CO}_{2}$ & $51+14$ \\
\hline $\mathrm{V}^{\prime} \mathrm{O}_{2} / \mathrm{HR} \mathrm{mL} \cdot$ beat $^{-1}$ & $7.4 \pm 2.2$ \\
\hline Borg scale (leg fatigue) & $7 \pm 2$ \\
\hline Borg scale (dyspnoea) & $7 \pm 2$ \\
\hline
\end{tabular}

Data are presented as $n$ or mean \pm SD. PAH: pulmonary arterial hypertension; IPAH: idiopathic PAH; PAH-Her: heritable PAH; PAH-CTD: PAH associated with connective tissue disease; PAH-CHD: PAH associated with congenital heart disease; BMI: body mass index; WHO: World Health Organization; RAP: right atrial pressure; PAP: pulmonary arterial pressure; PCWP: pulmonary capillary wedge pressure; PVRI: pulmonary vascular resistance index; WU: Wood units; 6MWD: 6-min walking distance; \% pred: \% predicted; CPET: cardiopulmonary exercise test; HR: heart rate; $V^{\prime} \mathrm{O}_{2}$ : oxygen uptake; RER: respiratory exchange ratio; $V^{\prime} \mathrm{E}$ : minute ventilation; $\mathrm{SpO}_{2}$; arterial oxygen saturation measured by pulse oximetry; $V^{\prime} E / V^{\prime} \mathrm{CO}_{2}$ : ventilatory equivalent for carbon dioxide; $V^{\prime} \mathrm{O}_{2} /$ HR: oxygen pulse. ${ }^{\#}$ : includes two patients with Eisenmenger physiology related to persistent arterial canal and ventricular septal defect. Predicted values are from CASANOva et al. [15].

associated with connective tissue disease and were in functional class II (75\%). They were treated with bosentan $(n=15)$, ambrisentan $(n=3)$ and epoprostenol i.v. $(n=2)$ for $22 \pm 18$ months.

\section{Test-retest repeatability}

Test-retest repeatabilities and corresponding Bland-Altman plots are shown in figure 3. Following 4 weeks of placebo, the mean \pm SD differences in the duration/length of the 6MWT, the ESWT and the CET were $-5 \pm 22 \mathrm{~m},+19 \pm 144 \mathrm{~s}$ and $-14 \pm 35 \mathrm{~s}$, respectively. The $6 \mathrm{MWT}$ showed less variation than the ESWT and the CET, and appeared to be the most repeatable test, as assessed by the coefficients of variation (table 2), the Pearson correlations and the modified Bland-Altman analysis. The heart rate, oxygen uptake and the oxygen saturation by pulse oximetry were the most repeatable physiological parameters (table 2).

\section{Responsiveness of exercise tests}

Responsiveness of exercise tests is shown in figure 4. The placebo-corrected changes in exercise capacity following the addition of sildenafil citrate to the baseline therapy were $18 \pm 25 \mathrm{~m}(\mathrm{p}=0.02), 58 \pm 235 \mathrm{~s}$ $(\mathrm{p}=0.58)$ and $29 \pm 77 \mathrm{~s}(\mathrm{p}=0.09)$ for the $6 \mathrm{MWT}$, the ESWT and the CET, respectively. Despite relative increases in 6 MWT being lower compared with endurance tests $(+4 \%,+15 \%$ and $+12 \%$ from baseline), the standardised response mean $(95 \% \mathrm{CI})$ values were $0.72(0.23-1.21), 0.25(-0.20-0.70)$ and $0.38(0.07-0.83)$ for the $6 \mathrm{MWT}$, ESWT and CET, respectively. Cardiac and ventilatory responses at peak exercise were unchanged following the addition of sildenafil citrate (data not shown). The mean \pm SD Likert scores following sildenafil citrate and placebo were $0.5 \pm 1.4$ and $0.4 \pm 1.1, \mathrm{p}=0.77$, respectively. The Likert scores correlated with the objective changes in duration/length for the 6MWT $(r=0.32 ; p=0.05)$, the ESWT 

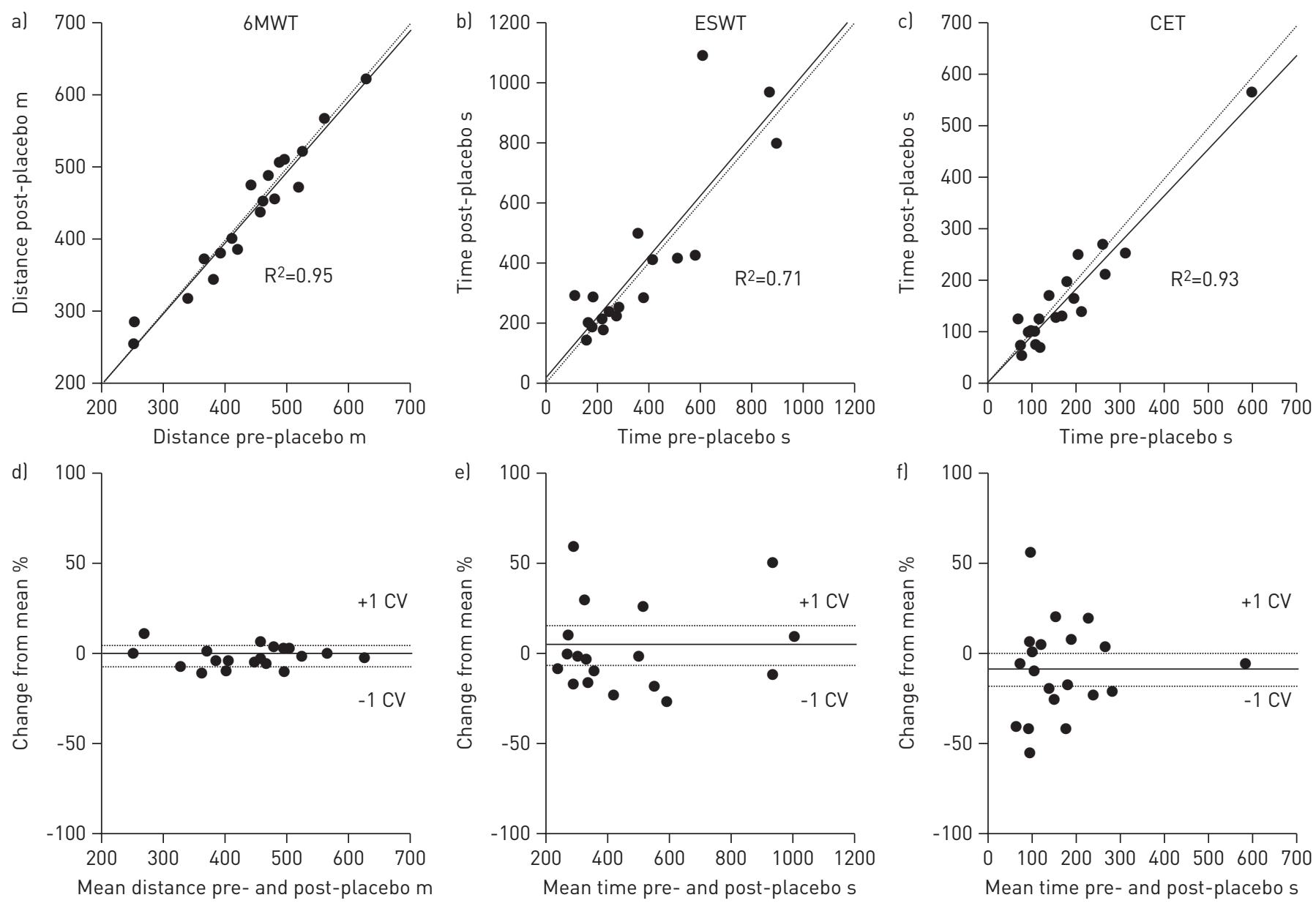

FIGURE 3 Test-retest repeatabilities of duration/length of a) the 6-min walk test (6MWT), b) the endurance shuttle walk test (ESWT) and c) the cycle endurance test (CET) are shown. The duration/length of post-placebo tests significantly correlated with pre-placebo tests for the 6MWT, the ESWT and the CET. The Pearson correlations (solid lines) and the identity lines (dotted lines) are shown. $\mathrm{d}-\mathrm{f}$ ) Modified Bland-Altman plot showing the relative change between pre- and post-placebo tests according to the mean duration/length of d) the 6MWT, e) the ESWT and f) the CET. The solid line represents the mean relative difference between the two tests, whereas the dotted lines correspond to the coefficients of variation (CV). The CV were similar for patients below and above the median value at baseline for the $6 \mathrm{MWT}(4 \pm 3 \%$ versus $2 \pm 2 \%)$, the ESWT $(12 \pm 13 \%$ versus $15 \pm 10 \%)$ and the CET ( $16 \pm 16$ versus $11 \pm 8 \%)$. The limits of agreement $(1.96 \mathrm{SD})$ were $-48-38 \mathrm{~m},-263-301 \mathrm{~s}$ and $-83-56 \mathrm{~s}$ for the 6MWT, the ESWT and the CET, respectively.

TABLE 2 Test-retest repeatability of the physiological parameters

\begin{tabular}{|c|c|c|c|c|c|c|c|c|c|c|c|c|}
\hline & \multicolumn{4}{|c|}{ 6MWT } & \multicolumn{4}{|c|}{ ESWT } & \multicolumn{4}{|c|}{ CET } \\
\hline & $\begin{array}{c}\text { Pre- } \\
\text { placebo }\end{array}$ & $\begin{array}{l}\text { Post- } \\
\text { placebo }\end{array}$ & $\begin{array}{c}\text { Pearson } \\
\mathbf{R}^{2}\end{array}$ & $\begin{array}{l}\text { CV } \\
\%\end{array}$ & $\begin{array}{c}\text { Pre- } \\
\text { placebo }\end{array}$ & $\begin{array}{l}\text { Post- } \\
\text { placebo }\end{array}$ & $\begin{array}{c}\text { Pearson } \\
\mathbf{R}^{2}\end{array}$ & $\begin{array}{l}\text { CV } \\
\%\end{array}$ & $\begin{array}{c}\text { Pre- } \\
\text { placebo }\end{array}$ & $\begin{array}{l}\text { Post- } \\
\text { placebo }\end{array}$ & $\begin{array}{c}\text { Pearson } \\
\mathbf{R}^{2}\end{array}$ & $\begin{array}{l}\text { CV } \\
\%\end{array}$ \\
\hline Distance m & $445 \pm 97$ & $440 \pm 98$ & 0.95 & 3 & $424 \pm 312$ & $452 \pm 342$ & 0.70 & 18 & NA & NA & NA & NA \\
\hline Time $\mathbf{s}$ & NA & NA & NA & NA & $373 \pm 236$ & $392 \pm 278$ & 0.71 & 14 & $179 \pm 121$ & $165 \pm 115$ & 0.93 & 13 \\
\hline HR beats $\cdot \min ^{-1}$ & $124 \pm 22$ & $126 \pm 22$ & 0.93 & 3 & $128 \pm 19$ & $127 \pm 20$ & 0.89 & 2 & $135 \pm 22$ & $135 \pm 23$ & 0.90 & 2 \\
\hline RER & $1.08 \pm 0.13$ & $1.05 \pm 0.11$ & 0.69 & 4 & $1.11 \pm 0.12$ & $1.10 \pm 0.12$ & 0.57 & 4 & $1.26 \pm 0.13$ & $1.25 \pm 0.13$ & 0.74 & 4 \\
\hline$V^{\prime} E \mathrm{~L} \cdot \min ^{-1}$ & $47 \pm 10$ & $48 \pm 10$ & 0.79 & 5 & $53 \pm 8$ & $53 \pm 8.11$ & 0.48 & 6 & $56 \pm 10$ & $56 \pm 12$ & 0.71 & 4 \\
\hline$V^{\prime} \mathrm{E}^{\prime} V^{\prime} \mathrm{CO}_{2}$ & $50 \pm 17$ & $52 \pm 20$ & 0.96 & 5 & $54 \pm 18$ & $53 \pm 18$ & 0.93 & 5 & $51 \pm 14$ & $51 \pm 15$ & 0.96 & 3 \\
\hline $\begin{array}{l}\mathrm{V}^{\prime} \mathrm{O}_{2} / \mathrm{HR} \\
\mathrm{mL} \cdot \text { beat }^{-1}\end{array}$ & $7.5 \pm 2.0$ & $7.6 \pm 2.4$ & 0.89 & 5 & $7.8 \pm 2.1$ & $7.8 \pm 2.0$ & 0.92 & 4 & $7.1 \pm 2.0$ & $7.0 \pm 2.1$ & 0.92 & 4 \\
\hline $\mathrm{SpO}_{2} \%$ & $85 \pm 8$ & $86 \pm 8$ & 0.97 & 1 & $86 \pm 7$ & $86 \pm 8$ & 0.85 & 2 & $86 \pm 9$ & $87 \pm 8$ & 0.80 & 2 \\
\hline
\end{tabular}

6MWT: 6-min walk test; ESWT: endurance shuttle walk test; CET: cycle endurance test; $\mathrm{CV}$ : coefficient of variation; $\mathrm{HR}$ : heart rate; $V^{\prime} \mathrm{O}_{2}$ : oxygen uptake; $V^{\prime} \mathrm{CO}_{2}$ : carbon dioxide production; RER: respiratory exchange ratio; $V^{\prime} \mathrm{E}$ : minute ventilation; $V^{\prime} \mathrm{E} / V^{\prime} \mathrm{CO}_{2}$ : ventilatory equivalent for carbon dioxide; $V^{\prime} \mathrm{O}_{2} / \mathrm{HR}$ : oxygen pulse; $\mathrm{SpO}_{2}$; arterial oxygen saturation measured by pulse oximetry; NA: not applicable. 

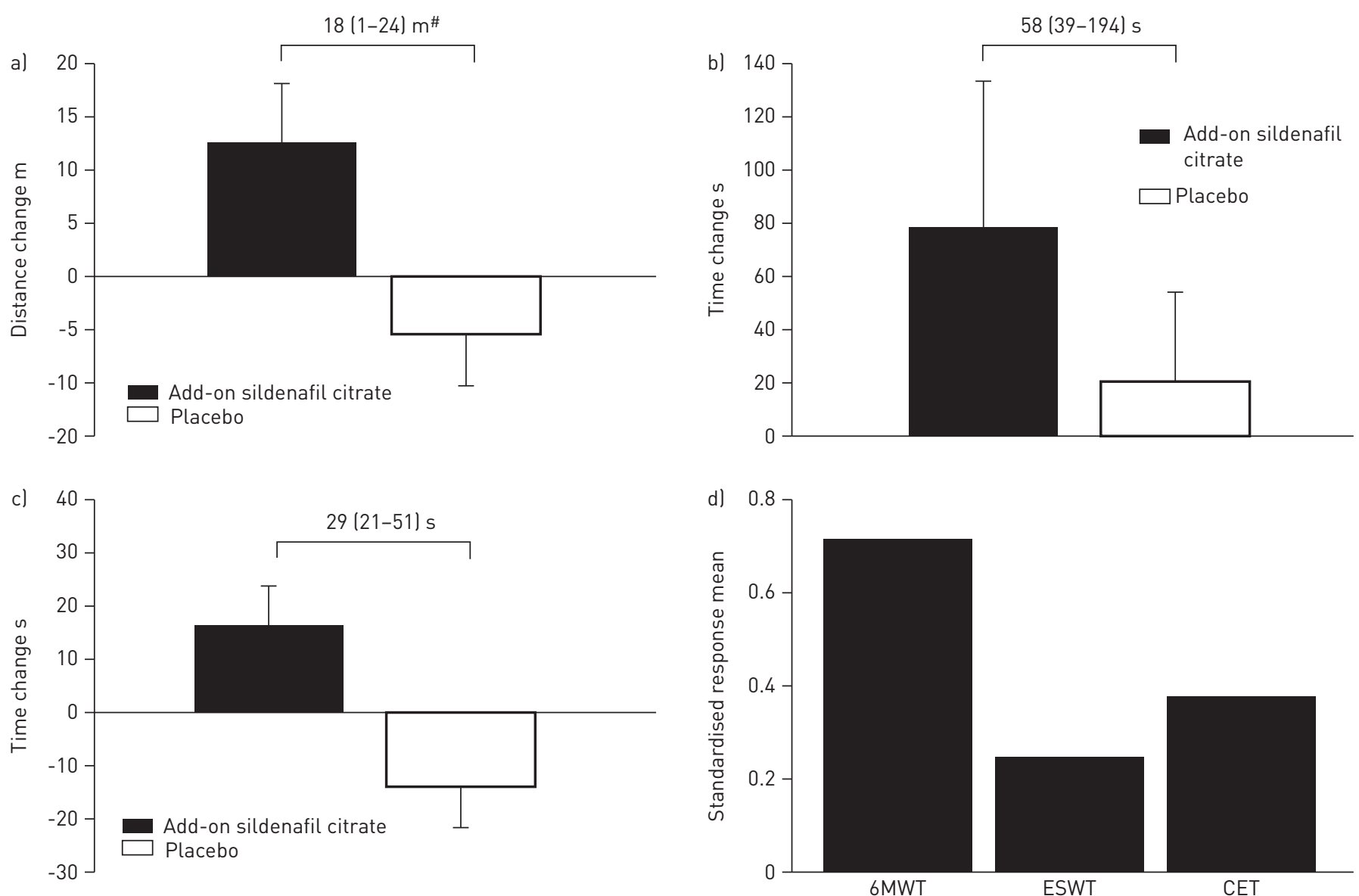

FIGURE 4 Responsiveness of a) the 6-min walk test (6MWT), b) the endurance shuttle walk test (ESWT) and c) the cycle endurance test (CET). Data are presented as mean change in duration/length following add-on sildenafil citrate and placebo, and the mean (95\% CI) placebo-corrected changes are given. $\mathrm{d}$ ) The standardised response mean of each test. ${ }^{\#}: \mathrm{p}=0.02$.

$(\mathrm{r}=0.52 ; \mathrm{p}=0.01)$ and the CET $(\mathrm{r}=0.35 ; \mathrm{p}=0.04)$, respectively. The areas under the ROC curve to detect a clinically perceptible improvement in exercise capacity (Likert score $\geqslant 1$ ) were $0.63,0.74$ and 0.63 for the 6MWT, the ESWT and the CET, respectively. The increase in exercise capacity associated with a true positive rate (specificity) of $90 \%$ to be associated with a Likert score $\geqslant 1$ were $32 \mathrm{~m}, 66 \mathrm{~s}$ and $53 \mathrm{~s}$ for the 6MWT, the ESWT and the CET, respectively.

\section{Discussion}

This study documented that among exercise tests currently used in chronic respiratory disorders, the 6MWT was the most likely to capture changes in exercise capacity induced by the addition of sildenafil citrate over baseline monotherapy in PAH. The enhanced responsiveness of the 6MWT was related to its better repeatability compared with the CET and the ESWT, supporting the use of this functional exercise test as an appropriate and clinically relevant end-point in randomised controlled trials.

Exercise capacity is generally considered to be a surrogate marker of disease severity in PAH [1]. Actually, the 6MWT and the CPET have excellent discriminative properties in this disease, and are closely correlated with functional class, disease severity and survival especially for idiopathic PAH patients $[1,16]$. However, their responsiveness has been questioned recently. Indeed, previous randomised controlled trials failed to show improvement in exercise capacity when the peak oxygen uptake assessed by CPET was the primary end-point [3] or when the 6MWT was the outcome measure in less severely impaired patients [17].

Previous studies concluded that the ESWT [7] or the CET [6] were more responsive than the 6MWT to bronchodilators in COPD. In PAH, the relative increase in exercise tolerance was enhanced when assessed using the CET compared with the 6MWT following exercise training $[18,19]$. In the present study, the relative increase in exercise capacity following the addition of sildenafil citrate was also higher for the ESWT and the CET compared with the 6MWT. However, only the increase in the 6MWT was statistically 
significant following the addition of sildenafil citrate to the baseline monotherapy. Using the standardised response mean, we also found that the ability to capture changes in exercise capacity was greater for the 6MWT than for the ESWT or the CET. Indeed, the standardised response mean for the 6MWT was close to 0.8 , a threshold considered "large" compared with values between 0.2 and 0.5 , which were considered to be small to moderate for the ESWT and the CET, respectively [20]. This parameter was chosen rather than the t-statistic, which provides no indication of random variation between tests, or the Cohen's effect size, which fails to recognise the relationship between placebo and treatment values. The standardised response mean is thus one of the most appropriate measures of the responsiveness of a measuring tool [21]. It has direct implication in the determination of sample size for clinical trials: the larger the standardised response mean, the smaller the sample size needed to demonstrate a treatment effect. The better repeatability of the 6MWT may account for its increased ability to capture changes in exercise capacity following the addition of sildenafil citrate. Indeed, the coefficient of variation of the $6 \mathrm{MWT}$ distance was well within $10 \%$, an arbitrary value considered to be optimal for this sort of assessment tool [14]. It is noteworthy that the enhanced repeatability of the 6MWT might have been influenced by the fact that this test is repeatedly performed by $\mathrm{PAH}$ patients as part of their routine follow-up. Indeed, exercise tests are associated with a learning effect [9]. However, special attention was made to ensure that any variability between the tests was related to random errors instead of systematic bias as patients underwent practice tests to minimise any learning effect. Also, the order of the tests for each subject was randomly determined. Moreover, systematic bias was excluded using Bland-Altman analysis and crossover statistical analyses did not reveal any sequence, visit or time effect. As the majority of patients were in WHO functional class II, it is also possible that most patients exercised near their maximal walking speed, which may have increased the 6MWT repeatability. Therefore, whether such repeatability is representative of its reliability in the setting of a multicentre study, with more severe patients and with a larger cohort of patients, remains unknown. However, the capacity of the 6MWT to document clinical improvement in previous randomised controlled trials supports this concept [2].

Very few studies have assessed the effect of a combination therapy on exercise capacity in PAH. When the previous randomised placebo-controlled trials were pooled in a recent meta-analysis, the mean increase in 6MWT was $22 \mathrm{~m}$ when a second PAH therapy was added to baseline therapy compared with placebo [4]. Only one study has assessed the effect of sildenafil citrate $80 \mathrm{mg}$ three times daily in addition to baseline epoprostenol i.v.; it found an improvement of 28.8 (95\% CI 13.9-43.8) $\mathrm{m}$ in the 6MWT distance with the combination therapy compared with epoprostenol i.v. alone [22]. In the present study, a relatively low dose of sildenafil citrate was used for a shorter period of time (4 versus 16 weeks) to assess responsiveness of exercise tests in $\mathrm{PAH}$, for the following reasons: 1) only sildenafil citrate $20 \mathrm{mg}$ three times daily is commercialised for the treatment of PAH in Canada; 2) in the pivotal randomised controlled trial evaluating the effect of sildenafil citrate monotherapy [23], most of the improvement in exercise capacity occurred during the first 4 weeks of treatment with no dose-effect relationship; and 3) 4 weeks of placebo was deemed to represent an appropriate period to assess mid-term repeatability of exercise testing while minimising the risk of clinical deterioration on placebo. Finally, a majority of our patients were in WHO functional class II. Therefore, the study design, potential drug-drug interaction between bosentan and sildenafil citrate [24] and patients' characteristics may have negatively affected the increase in exercise capacity observed in our study.

Previous studies have suggested that the minimal detectable difference (assessed using distribution-based method) [25] and the minimal important difference (assessed using anchor-based method) [26] of the $6 \mathrm{MWT}$ distance following the initiation of PAH therapy were $30-40 \mathrm{~m}$. Although significant, the improvement in walking distance observed following the combination of sildenafil citrate to baseline monotherapy was thus modest and below the suggested minimal important difference for this variable and for the perception threshold. However, changes in exercise capacity significantly correlated with the subjective perception of change in clinical status as assessed by a Likert scale. In an attempt to assess the minimal increase in exercise capacity that was perceptible by patients in our cohort, a ROC curve was constructed. Changes in 6MWT distance and in ESWT that had $>90 \%$ chance (specificity) of being perceived by the patient were close to the minimal important difference of the 6MWT previously suggested in PAH [26], as well as previously reported in COPD for the ESWT [27]. Conversely, the perception threshold for the CET was far from the proposed minimal important difference of $105 \mathrm{~s}$ reported for CET in COPD [28]. One potential explanation is that our CET was too short in duration. We arbitrarily chose to set the intensity at $80 \%$ of the peak workload to try to minimise the high variability that has been seen in COPD at lower relative workloads [29]. However, according to the American Thoracic Society, a constant workload test should last a minimum of $360 \mathrm{~s}$ [9], which was not the case in our study. The relatively high intensity might have induced early peripheral muscle acidosis and fatigue. Therefore, the CET might have been less susceptible to track a beneficial effect on pulmonary haemodynamics following sildenafil citrate 
administration. This also reinforces the fact that minimal important difference estimates obtained in one disease cannot necessarily be extrapolated to other conditions and that the minimal important difference might be disease, severity and intervention specific [30].

\section{Conclusion}

Amongst the exercise tests commonly used to evaluate patients with chronic respiratory diseases, the 6MWT had the best ability to capture changes in exercise capacity when sildenafil citrate was combined with patients' baseline monotherapy in PAH. This enhanced responsiveness was related to a better repeatability of the 6MWT compared with the ESWT and the CET. Changes in exercise capacity also correlated with changes in patients' perception of exercise tolerance. These results support the use of the $6 \mathrm{MWT}$ as an outcome measure, especially in the setting of controlled trials in PAH.

\section{Acknowledgements}

The authors acknowledge Serge Simard for statistical analysis and Luce Bouffard for technical assistance (Centre de recherche de l'Institut Universitaire de cardiologie et de pneumologie de Québec, Université Laval, Québec, QC, Canada).

\section{References}

1 Miyamoto S, Nagaya N, Satoh T, et al. Clinical correlates and prognostic significance of six-minute walk test in patients with primary pulmonary hypertension. Comparison with cardiopulmonary exercise testing. Am J Respir Crit Care Med 2000; 161: 487-492.

2 Galie N, Manes A, Negro L, et al. A meta-analysis of randomized controlled trials in pulmonary arterial hypertension. Eur Heart J 2009; 30: 394-403.

3 Barst RJ, McGoon M, McLaughlin V, et al. Beraprost therapy for pulmonary arterial hypertension. J Am Coll Cardiol 2003; 41: 2119-2125.

4 Bai Y, Sun L, Hu S, et al. Combination therapy in pulmonary arterial hypertension: a meta-analysis. Cardiology 2011; 120: 157-165.

5 Frost AE, Langleben D, Oudiz R, et al. The 6-min walk test (6MW) as an efficacy endpoint in pulmonary arterial hypertension clinical trials: demonstration of a ceiling effect. Vascul Pharmacol 2005; 43: 36-39.

6 Oga T, Nishimura K, Tsukino M, et al. The effects of oxitropium bromide on exercise performance in patients with stable chronic obstructive pulmonary disease. A comparison of three different exercise tests. Am J Respir Crit Care Med 2000; 161: 1897-1901.

7 Pepin V, Brodeur J, Lacasse Y, et al. Six-minute walking versus shuttle walking: responsiveness to bronchodilation in chronic obstructive pulmonary disease. Thorax 2007; 62: 291-298.

8 Galie N, Hoeper MM, Humbert M, et al. Guidelines for the diagnosis and treatment of pulmonary hypertension. Eur Respir J 2009; 34: 1219-1263.

9 American Thoracic Society, American College of Clinical Pharmacy. ATS/ACCP statement on cardiopulmonary exercise testing. Am J Respir Crit Care Med 2003; 167: 211-277.

10 Borg GA. Psychophysical bases of perceived exertion. Med Sci Sports Exerc 1982; 14: 377-381.

11 American Thoracic Society. ATS statement: guidelines for the six-minute walk test. Am J Respir Crit Care Med 2002; 166: 111-117.

12 Singh SJ, Morgan MD, Scott S, et al. Development of a shuttle walking test of disability in patients with chronic airways obstruction. Thorax 1992; 47: 1019-1024.

13 Revill SM, Morgan MD, Singh SJ, et al. The endurance shuttle walk: a new field test for the assessment of endurance capacity in chronic obstructive pulmonary disease. Thorax 1999; 54: 213-222.

14 Atkinson G, Nevill AM. Statistical methods for assessing measurement error (reliability) in variables relevant to sports medicine. Sports Med 1998; 26: 217-238.

15 Casanova C, Celli BR, Barria P, et al. The 6-min walk distance in healthy subjects: reference standards from seven countries. Eur Respir J 2011; 37: 150-156.

16 Wensel R, Opitz CF, Anker SD, et al. Assessment of survival in patients with primary pulmonary hypertension: importance of cardiopulmonary exercise testing. Circulation 2002; 106: 319-324.

17 Galie N, Rubin LJ, Hoeper M, et al. Treatment of patients with mildly symptomatic pulmonary arterial hypertension with bosentan (EARLY study): a double-blind, randomised controlled trial. Lancet 2008; 371: 2093-2100.

18 de Man FS, Handoko ML, Groepenhoff $\mathrm{H}$, et al. Effects of exercise training in patients with idiopathic pulmonary arterial hypertension. Eur Respir J 2009; 34: 669-675.

19 Mainguy V, Maltais F, Saey D, et al. Effects of a rehabilitation program on skeletal muscle function in idiopathic pulmonary arterial hypertension. J Cardiopulm Rehabil Prev 2010; 30: 319-323.

20 Liang MH, Fossel AH, Larson MG. Comparisons of five health status instruments for orthopedic evaluation. Med Care 1990; 28: 632-642.

21 Norman GR, Wyrwich KW, Patrick DL. The mathematical relationship among different forms of responsiveness coefficients. Qual Life Res 2007; 16: 815-822.

22 Simonneau G, Rubin LJ, Galie N, et al. Addition of sildenafil to long-term intravenous epoprostenol therapy in patients with pulmonary arterial hypertension: a randomized trial. Ann Intern Med 2008; 149: 521-530.

23 Galie N, Ghofrani HA, Torbicki A, et al. Sildenafil citrate therapy for pulmonary arterial hypertension. $N$ Engl J Med 2005; 353: 2148-2157.

24 Paul GA, Gibbs JS, Boobis AR, et al. Bosentan decreases the plasma concentration of sildenafil when coprescribed in pulmonary hypertension. Br J Clin Pharmacol 2005; 60: 107-112.

25 Gilbert C, Brown MC, Cappelleri JC, et al. Estimating a minimally important difference in pulmonary arterial hypertension following treatment with sildenafil. Chest 2009; 135: 137-142.

26 Mathai SC, Puhan MA, Lam D, et al. The minimal important difference in the 6-minute walk test for patients with pulmonary arterial hypertension. Am J Respir Crit Care Med 2012; 186: 428-433. 
27 Pepin V, Laviolette L, Brouillard C, et al. Significance of changes in endurance shuttle walking performance. Thorax 2011; 66: 115-120.

28 Casaburi R. Factors determining constant work rate exercise tolerance in COPD and their role in dictating the minimal clinically important difference in response to interventions. COPD 2005; 2: 131-136.

29 Neder JA, Jones PW, Nery LE, et al. Determinants of the exercise endurance capacity in patients with chronic obstructive pulmonary disease. The power-duration relationship. Am J Respir Crit Care Med 2000; 162: 497-504.

30 Quittner AL, Modi AC, Wainwright C, et al. Determination of the minimal clinically important difference scores for the Cystic Fibrosis Questionnaire - revised respiratory symptom scale in two populations of patients with cystic fibrosis and chronic Pseudomonas aeruginosa airway infection. Chest 2009; 135: 1610-1618. 